entirely appropriate to their stage of development), but that they did not recognise them as models. To imagine that probability has any greater claim to inherent permanency than mechanisms-and, in particular, to draw fundamental conclusions from the accident that probability suggests an intrinsic uncertainty-is to make the same error.

It seems likely that the quantum theory, so far from expelling precision from our description of Nature, really opens the door to it for the first time. For strictly speaking, a field theory can never allow absolute precision since continua are infinitely divisible. A particle having a co-ordinate represented by a non-terminating decimal, for example, could have its position specified as nearly exactly as we pleased, but not with absolute exactness, and an infinite future would hold the possibility of an indefinite amount of departure from a prediction based on such specification. Data which must necessarily be expressed in integers, however, are clearly susceptible of absolutely exact expression. The present position is therefore that we have escaped from a scheme of thought which made precise prediction impossible into one which, though we are as yet less far advanced in it, offers absolute precision as a possible goal.

Comparisons, if not odious, are liable to be misleading, and it would be unwise to stress them. Nevertheless, it may well be doubted whether in any previous period of twenty-five years, physics has experienced a more substantial forward movement.

\title{
Constitution of the Earth
}

By Dr. Harold Jeffreys, F.R.S., St. John's College, Cambridge

COMPARING the position of geophysics now with what existed in 1910, while we are struck by the great development that has taken place, we are equally struck, on looking more closely, by the fact that most of the theoretical advances are due, not to specifically new methods, but to the fuller application of methods that were already known. The work of Kelvin and Sir George Darwin on the rigidity of the earth, and on the evolution of the earth-moon system under the action of tidal friction, was already classical; Darwin's theory of the stresses needed to support continents and mountains was thirty years old; the existence of isostatic compensation, and the two alternative explanations of Pratt and Airy, had been known for fifty years, Stokes's theory of the determination of the figure of the earth from observations of gravity for sixty, and Poisson's theory of the longitudinal and transverse waves in an elastic solid for eighty. Dr. C. Davison, still with us, had put the thermal contraction theory of mountain formation on a quantitative basis in 1887, and Wiechert had shown how to reconcile the earth's ellipticity and precessional constant on the assumption of a thick rocky shell surrounding a dense metallic core. The existence of a change in properties in the crust in the continents at some small depth had already been inferred from geological considerations by Suess.

The chief new advance in the first ten years of the present century probably arose from the detection of radioactivity, the recognition of its effect in modifying the earth's thermal history, and the use of the rate of disintegration of uranium to find the absolute ages of minerals and to calibrate the geological time-scale. The age of the earth, estimated from thermal considerations by Kelvin at about 20 million years, was suddenly raised to about 1,500 million. Physicists did not all accept the new estimate without a struggle, though purely mechanical considerations might have given some ground for doubting Kelvin's value. Darwin, by adopting such a viscosity in the earth as would make the changes through tidal friction occur at every time at the maximum rate, the viscosity thus varying with time in a way very unlikely to correspond to the facts, could not bring the age of the moon below 54 million years. This might have been taken as an absolute minimum that was practically certain to be greatly exceeded.

The new source of heat was so potent that the present Lord Rayleigh pointed out that, if it was not confined to a depth of some tens of kilometres at the outside, it would produce more heat than is escaping from the earth; consequently it led Holmes to estimate the rate of decrease with depth. It was found to suggest that average granite could exist only to a depth of about $15 \mathrm{~km}$. and agreed in principle with the conclusions of Suess.

Meanwhile, seismology made three great advances. Herglotz and Bateman provided a method of finding the velocity of an elastic wave at any depth in the earth from the observed times of travel of earthquake waves, which was first applied by S. Mohorovičić in 1916. R. D. Oldham found that longitudinal waves arrived at the 
opposite side of the earth about three minutes later than they would if the velocities found from observations at shorter distances were maintained to the centre, and inferred that at a depth of about half the radius there was a change of properties involving a diminution of velocity. The radius found for the core in this way was substantially less than that found by Wiechert; but it was not until 1926 that it was noticed that the compression of each layer in the earth by the weight of the matter above it would raise the density so much that, when it was allowed for, the radii found by Wiechert's method and Oldham's were practically identical, so that the Wiechert core and the Oldham core are the same thing. A. Mohorovičić, working on a small earthquake in Croatia in 1909, found that the records at short distances could not be represented by a medium the properties of which varied continuously. They showed a pair of strong longitudinal and transverse waves, which were overtaken at a distance between $100 \mathrm{~km}$. and $200 \mathrm{~km}$. by a weaker pair that travelled faster; the latter corresponded to the waves observed at greater distances. The interpretation is that the strong pair observed only up to distances of about $800 \mathrm{~km}$. travel in an upper layer, while the others travel with greater velocities through a lower region of great depth. The correspondence with the geological and thermal considerations is plain.

All these lines of investigation were greatly developed by Gutenberg, particularly in relation to the waves through the core and reflected by it. The reduction of the velocity of a longitudinal wave on entering the core is about that of light on entering water from air ; consequently the core casts a shadow, which can be recognised by the absence of the clear direct waves beyond a distance of about $105^{\circ}$ and their replacement by a vague diffracted movement, and the rays passing through it have a caustic surface, which meets the outer surface before it has come to a focus. Thus there is a narrow zone, at a distance of about $143^{\circ}$, where the motion in the longitudinal wave is extremely strong. The estimated velocities led Gutenberg to calculate times of transmission of many other core waves, notably those reflected at its outside, the wave that is transverse in the shell but is partly refracted as a longitudinal one in the core, and one derived from the latter by undergoing one reflexion on the inside of the core before it comes out. These were all recognised on actual seismograms. It was much later, however, that attention was directed to this work in Great Britain, and Prof. H. H. Turner rediscovered several of Gutenberg's waves independently from the observational material supplied to the International Seismological Summary.
The most immediate consequence of the existence of the reflected waves is that the boundary of the core is a sharp discontinuity of material and not a gradual transition. This gives a direct verification of the inference, based on a slightily dubious analogy with meteorites, that the shell is stony and the core mainly iron, with probably a certain amount of nickel. Further, the mean densities found by combining the radius of the core with the mean density and the moment of inertia are about 4.5 and 12 respectively; but if we estimate what they would become if the high pressures were taken off, they are about $3 \cdot 3$ and 8 , the former agreeing with the density of olivine and the latter with that of iron. The core appears to be liquid. Gutenberg calculated what the times of transmis. sion of transverse waves through it would be if it was solid, but though several workers have found movements that they have thought to be these waves, their results are not consistent and seem to be capable of other interpretations. The most direct evidence on the state of the core is provided by the earth's tidal yielding, which is practically what it would be if the core was fluid, and sub. stantially more than if the core was solid and had a rigidity in any reasonable ratio to its bulk-modulus.

Further work on the records of near earthquakes has shown that there is at least one intermediate layer, and there may be three. The thickness of the upper layer is $12 \mathrm{~km}$., with an uncertainty of $3-4 \mathrm{~km}$.; the intermediate ones together may be twice as thick. The study of the surface waves, mainly by Stoneley, has given similar results with a rather higher precision. The surface. waves under the oceans, however, show a different structure; the thickness of the upper layer is substantially less, and the geological evidence indicates that it is not granite there, but probably andesite or even basalt.

The chief modern contributions to seismological technique are probably the wireless time service and Bridgman's invention of a method of experimenting at high pressures. Until recently, the time had to be determined independently by astronomical observation at every station ; now a station with no astronomical equipment can fix its time with as great accuracy as was possible to the best in 1910. The result of this and of the increase in the number of stations is that the times of transmission of the various waves can be determined, in most cases, with an accuracy of a second or less ; in fact, the accuracy is so high that it has become necessary to allow for the ellipticity of the earth before we can make full use of it, and it has only been attainable because the earthquakes used have been in much the same latitude, so that the effect of the ellipticity has always been nearly the same. 
Testing the compressibility of actual rock specimens at high pressures has shown that the velocities of elastic waves in the upper layer are consistent with its being granite; geologists seem to be coming to regard the upper layer as more like a granodiorite than a normal granite, but this is a minor change. The lower layer fits olivine or dunite in elasticity as well as in density; it is definitely too dense and too stiff to be basalt, which, if it forms any extended layer at all, can only be the deepest and least clearly recognisable of the intermediate ones.

A strong curvature near $20^{\circ}$ of the curves representing times of transmission against distance was first noticed by Byerly, and work by $\mathrm{I}$. Lehmann, K. E. Bullen and myself has shown that there is a sharp change in the slope there. This appears to correspond to an increase of the velocity by about 10 per cent at a depth of about $350 \mathrm{~km}$. The nature of this change is not yet understood. Apart from the upper layers, this discontinuity, and the boundary of the core, there are no other sudden changes in properties with depth. Search has been made for a sulphide layer, which has been expected to form the outermost part of the core, but it is necessary to do some violence to the observations to fit one in at all, and there seems to be no room for one more than a few kilometres thick at the most.

The study of gravity made a great advance in 1912 with the publication of Hayford's work in the United States, which showed that the larger mountain ranges of the United States are asso. ciated with such a defect of density below that the whole produces little disturbance of gravity. Unfortunately this work, and the later work of Bowie, have suffered greatly from exaggeration and misinterpretation. The general result was to assume that this compensation made a great reduction in the differences between observed and calculated gravity; but it did not abolish them. It was inferred by many that the approximate compensation was exact, and elaborate theories have been constructed upon it, assuming that it showed not only the lower layer, but even the upper ones, to be completely devoid of strength, in direct opposition to the plain fact that the surface of the earth is not perfectly flat. Others, unwilling to accept the conclusion, have gone to the opposite extreme and denied that the observations imply any compensation at all. It still does not seem to be generally recognised that a theory that reduces the average residual in a mountainous region from twenty times to six times the probable error of a single observation, is on a different footing both from a theory that reduces it to the mean error of a single observation and from one that does not reduce it at all. On the other hand, the generality of the American results is not complete ; they seem to apply to all the great mountain regions where they have been tested, but they break down in India and in the East Indies, as De Graaff Hunter and Vening Meinesz have shown.

Meinesz's introduction of a method of determining gravity at sea by observing in a submarine is perhaps the greatest advance towards determining the figure of the earth accurately that has been made recently. Stokes showed how a complete knowledge of gravity over the earth's surface could give a determination of the external field; but so long as observations were available only over the land, and very limited proportions of that, we were in the position of trying to locate one end of a rod of unknown and variable curvature by observing a lot of points near the other. Now lines of observed values of gravity are available right across the main oceans, though there is still a great need for more in the southern hemisphere.

\section{The Measurement of Geological Time}

\section{By Prof. Arthur Holmes, Professor of Geology, University of Durham}

$\mathrm{T}^{\mathrm{w}}$ WENTY-FIVE years ago, opinions as to the scale of geological time were still in a chaotic state. The earlier controversy between Kelvin and the geologists had come to a dramatic end in 1906 with the discovery by Strutt (the present Lord Rayleigh) of the widespread distribution of radioactive elements through the rocks of the earth's crust. The earth could no longer be regarded as a spendthrift living on a limited capital of ancestral heat. An independent source of income had been disclosed in the energy liberated during radioactive disintegration, and henceforth no thermal argument could set a limit to the age of the earth.
Already, however, helium and lead had been recognised as the end-products of the uranium family, and Rutherford had suggested (1905 and 1906) that the accumulation of these elements in radioactive minerals might provide a measure of the age of such minerals.

In 1907 Boltwood made the first attempt to calculate the ages of minerals which had been analysed for uranium and lead. During the next three years, Strutt carricd out his far-reaching researches on the accumulation of helium during geological time and on its rate of production in uranium and thorium minerals. Thus, by 1910 